\title{
Spatial Decoupling of Light Absorption and Scattering Centers in Plasmon-assisted Bubble Column Evaporator for Solar Steam
}

\section{Generation}

\author{
Guohua Liu, ${ }^{1,2 *}$ Guansheng Yao, ${ }^{1}$ Jinliang $\mathrm{Xu}^{1,2}$ and Xin $\mathrm{Yan}^{1}$
}

\begin{abstract}
Solar water evaporation offers a promising route to enable large-scale conversion and storage of solar energy. Utilizing nanofluid with superior photothermal properties in solar energy systems is an effective method to improve their thermal performance. However, the bulk temperature increase of fluid will induce thermal loss, which reduces the phase-change evaporation at air-water interface. Here, we demonstrate a plasmon-assisted bubble column evaporator that functionally deconvolutes its light absorption and scattering centers for solar steam generation. Varying the gas fraction of bubbles and their surrounding particle-dispersed liquid marbles makes it possible to examine the interplay between the light absorptive nanoparticles and the scattering properties along bubble-liquid interfaces. This particle-stabilized gas/liquid dispersion optimizes the contributions of light harvesting, heat management and mass transport to the overall device performance, leading to a three-fold enhancement of evaporation under two-sun irradiation. Our findings pave a new way to enhance evaporation in nanofluid suspensions and speed the development of solar thermal system for practical applications.
\end{abstract}

Keywords: Solar energy; Nanofluid; Bubble column; Plasmonic heating; Evaporation.

Received: 2 March 2020; Accepted: 3 April 2020.

Article type: Research article.

\section{Introduction}

Half of the solar energy received on Earth's surface drives evaporation, fueling the global water cycle that affects water resources, ecosystems, weather and climate. ${ }^{[1-4]}$ As a fundamental photothermal process, solar water evaporation plays a ubiquitous role in diverse industries such as clean water, power generation, absorption chillers, desalination and steam sterilization. ${ }^{[5,6]}$ Water evaporation is a surface process, in which water molecules at the thin air-water interface in liquid side driven by their high energy state are transported into the vapor phase. ${ }^{[5,7,8]}$ While in nature, the solar radiation penetrates water through a certain depth depending on water quality and the light spectrum wavelength, and the water profile over light passage length is heated up homogeneously. ${ }^{[9]}$ Therefore, this nature's way of

\footnotetext{
${ }^{1}$ Beijing Key Laboratory of Multiphase Flow and Heat Transfer for Low Grade Energy Utilization, North China Electric Power University, Beijing, 102206, China.

${ }^{2}$ Key Laboratory of Power Station Energy Transfer Conversion and System, North China Electric Power University, Ministry of Education, Beijing 102206, China

*Email: liuguohua126@126.com (G. Liu)
}

evaporation is inefficient because it falls into the bulk heating where the water is uniformly heated up, which unavoidably results in heat loss due to the heat transfer to the bulk water.

Significant efforts have been directed toward improving the evaporation. Fluids seeded with nanoparticles in a volumetric dispersion have been earlier introduced to enhance evaporation because it can be facilely implemented with improved thermal conductivity. ${ }^{[10-12]}$ Modulating the light absorbers by changing the shape or composition of nanoparticles leads to the increased cross-section or wavelength of light absorption. ${ }^{[13-18]}$ However, bulk heating still exists in the suspension when the light is absorbed deep into bulk liquid. To minimize the heat loss, localizing the energy at air-water interface is a feasible way to improve the efficiency. ${ }^{[5,19-21]}$ This approach selectively heats the evaporative water rather than the entire body of water by a floating photothermal structure. Such interfacial heating system confines the heat at air-water interface that suppresses heat loss to the bulk water, but requires the special design of photothermal system to attain this purpose. ${ }^{[22-26]}$ Bubble column humidification is an industrial technology for seawater desalination, in which the carrier gas is dispersed into water column through a porous sinter to form small 
bubbles. ${ }^{[27,28]}$ The enormous number of unsaturated small bubbles offer a large interfacial area to enhance the heat and mass transfer, hence raising the gas humidity. ${ }^{[29-31]}$ Nevertheless, the use of bubble column humidifiers is very limited in solar water evaporation.
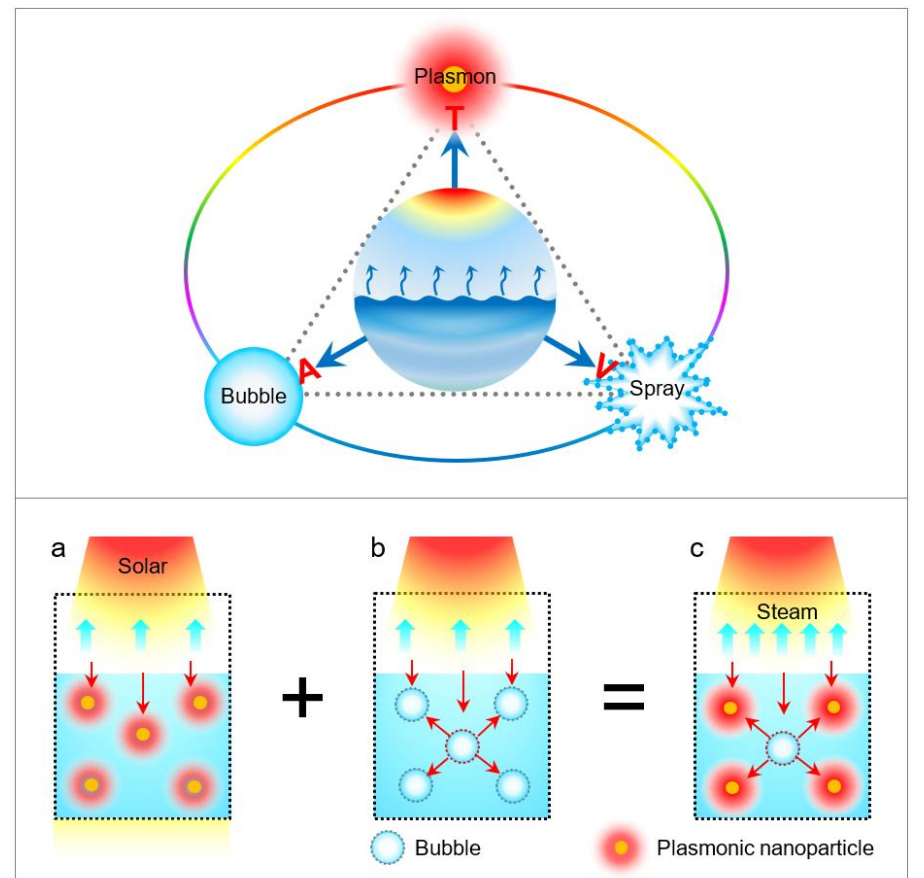

Fig. 1 The physical advantages and concept of plasmon-assisted bubble column evaporator. a) The characteristics of volumetric plasmon heating. b) Multiple light scattering from the dispersed bubbles. c) Spatial separation of light absorption and scattering concept for enhanced solar steam generation.

Most system optimizations are made from a single aspect of enhancing light harvesting, reducing heat loss or providing enough surface area for fast evaporation. While evaporation is principally governed by the surface energy balance between net radiation and heat losses due to turbulent convection and evaporation. ${ }^{[32-35]}$ It thus can be optimized via rational balance of the physical parameters including temperature $\mathrm{T}$, surface area $\mathrm{A}$ and convection velocity $\mathrm{V}$ (Fig. 1). Here, we, for the first time, report a proof-of-concept of plasmon-assisted bubble column evaporator for solar steam generation. This is realized by employing a multiphasic solution that is composed of light absorptive gold nanoparticles (Au NPs, Fig. 1a) and dynamic light scattering bubbles (Fig. 1b) to save the use of expensive Au NPs. With the mixing solution, multi-scattering is initiated over the whole column and the incident light is scattered in all directions and reabsorbed by the surrounding Au NPs (Fig. 1c). Such system with separated functions enabled us to finely tune the plasmon heating, bubble scattering and spray process to optimize the performance of localized evaporation and humidification, leading to a three-fold enhancement of the evaporation rate with a gas fraction of air $40 \mathrm{~mL} / \mathrm{min}$ into solution of Au NPs $50 \mathrm{ppm}$. This unique strategy coupling of solar evaporation and humidification is of importance for the development of new solar steam technologies.
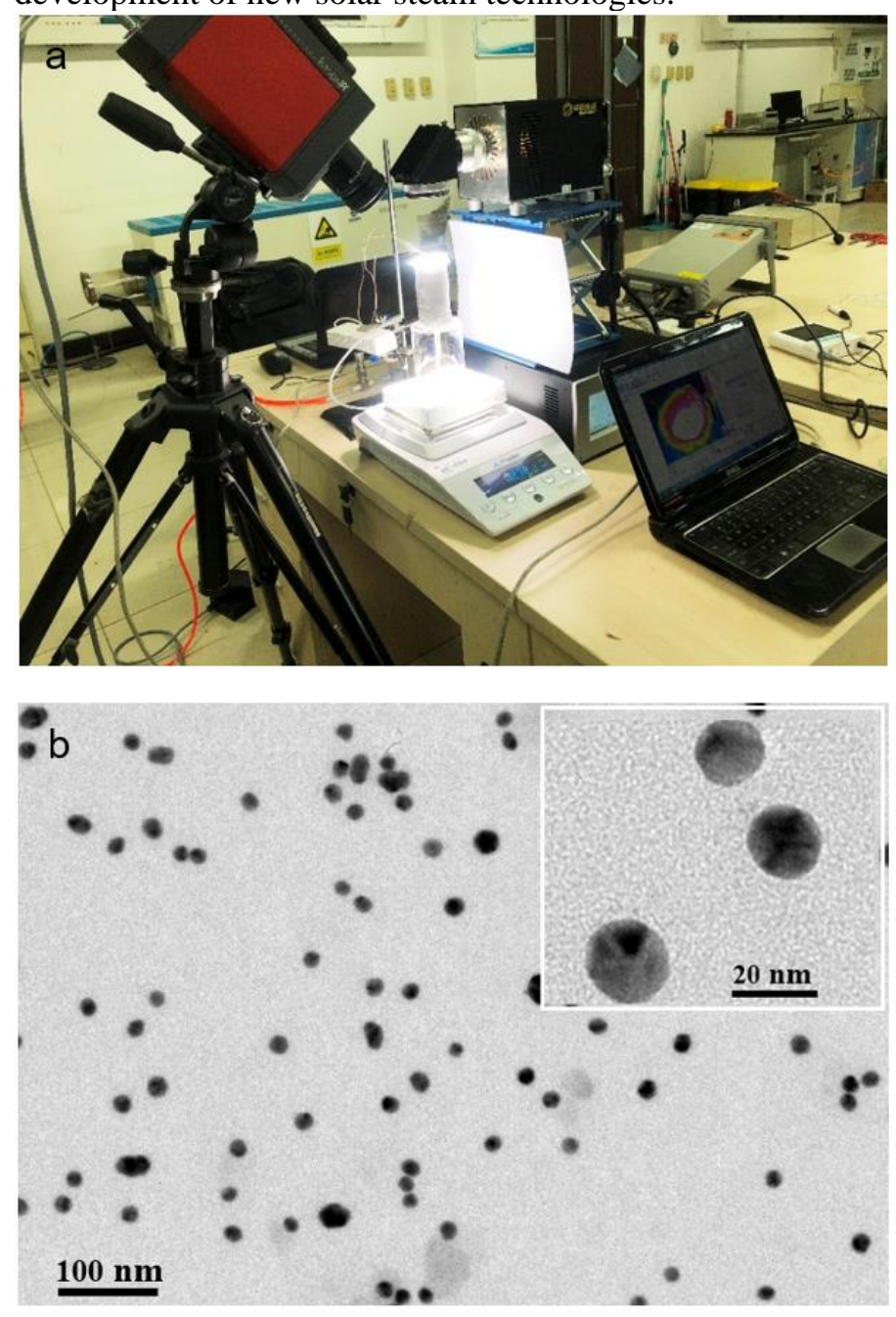

Fig. 2 a) A photograph of experimental set up, and b) a TEM image of gold nanoparticles with a mean size of $16.5 \mathrm{~nm}$.

\section{Experimental section}

The experimental setup is consisted of a solar simulator (CEL-PE300-3A) with a standard AM1.5filter, an electronic balance (Precisa LS/LT/LX 620M, $0.1 \mathrm{mg}$ accuracy), a highpressure gas cylinder, a pressure regulator, a needle valve (WL94H-64P) and a rotameter (LZB-2), a specially designed glass column (40 mm inner diameter and $100 \mathrm{~mm}$ height) integrated with a steel sinter $(13 \mathrm{~mm}$ diameter, $25 \mathrm{~mm}$ height and a pore size of $2 \sim 6 \mu \mathrm{m})$, three K-type thermocouples (Omega TT-T-36), a data acquisition model (Agilent 34970A), a high speed camera (IDT Motion Pro Y4, USA), an infrared camera image system (IR 5300 Series, Germany, spatial resolution of 320 pixels by 256 pixels) and a computer (Fig. 2a).

A high surface area of bubble-liquid interface was continuously produced by flowing air through the porous sinter into the glass column filled with the solution to a height of $85 \mathrm{~mm}$. The flow pattern with dispersed bubbles was analyzed by Image $\mathbf{J}$ and Image-Pro software to determine the primary bubble size at $0.6 \mathrm{~mm}$. The 
morphology of porous sinter was characterized using a SEM (JEOL FEG JSM 7001F) and the analysis showed the dispersion of pore size in range of 2-6 $\mu \mathrm{m}$. The synthesis of $\mathrm{Au}$ NPs followed standard methods detailed in previous work. ${ }^{[36]}$ The prepared nanofluids has good stability and can be diluted into the required concentration. The nanoparticles were characterized by a transmission electron microscopy (TEM-JEOL 2011) and the high-resolution image (Fig. 2b) exhibited a quasi-spherical morphology. The mean diameter was evaluated as $16.5 \mathrm{~nm}$ with a relative standard deviation of $8.2 \%$.

To evaluate the light scattering effect, the bubbly flow was illuminated by a green laser beam (Laser 303) in dark room. The optical scattering images were transiently recorded by a camera. The camera was mounted at a scattering angle of $90^{\circ}$ to capture the scattering intensity from bubbles due to reflection. The absorption spectra of plasmon-bubble nanofluids were examined by a UV-vis spectrophotometer (UV3600, Shimadzu, Japan) at room temperature. To simulate the bubbly flow in quartz cuvettes, small amount of an effervescent tablet in the solution was added to sustainably release carbon dioxide bubbles as a control experiment and the reference fluid was deionized (DI) water.

The evaporation experiments were conducted at an ambient temperature of $20{ }^{\circ} \mathrm{C}$ and humidity of $35 \%$. Their radiation density at level of air-water interface was fixed at 2 $\mathrm{kW} / \mathrm{m}^{2}$. The released pressure of gas cylinder was set at 20 $\mathrm{kPa}$. Prior to any test, gas leakage test was performed to avoid any leakage from the system. The air flow rate through the sinter was adjusted by the control valve. Each run is initiated when a steady condition is achieved. In the test, the external surface of column was insulated by glass wool blanket of $2.0 \mathrm{~cm}$ thickness to reduce the heat loss. The solution temperature was traced by the thermocouples (placed on top, middle and bottom of the solution). The surface temperature at steam-water interface was mapped by the infrared camera at speed of $1 \mathrm{~Hz}$ and the flow pattern was recorded by the high-speed camera at speed of $1000 \mathrm{~Hz}$. The balance was used to monitor the mass loss from evaporation in real time, recorded by the computer with RS-232 serial ports, and then used to determine the evaporation rate.

\section{Results}

\subsection{Optical properties}

When light interacts with an ensemble of randomly dispersed bubbles in water, the light can be scattered in all directions depending on the size and shape of rising bubbles. ${ }^{[37]}$ Fig. 3a shows the transient light-scattering images of the dynamic bubbly flow. The light beam was produced by a laser with a spot size of $2.0 \mathrm{~mm}$ and a wavelength of $532 \mathrm{~nm}$. In the first image, bubbles outside the light beam are nearly invisible and only several bubbles near the light beam can be identified (Fig. 3a 1-4-5). As the evolution of bubbly flow, some bubbles passing through the light beam induce strong reflection of the incoming light at all direction over the rising bubble surfaces. These reflections then sequentially transfer to the surrounding bubbles and recycle the reflection over the whole domain, leading to a multiple scattering effect in the bubble swarm. As a consequence, the scattering intensity due to the inner reflection between bubbles is very strong, illuminating the whole volume of column. (Fig. 3a 2-3-6).
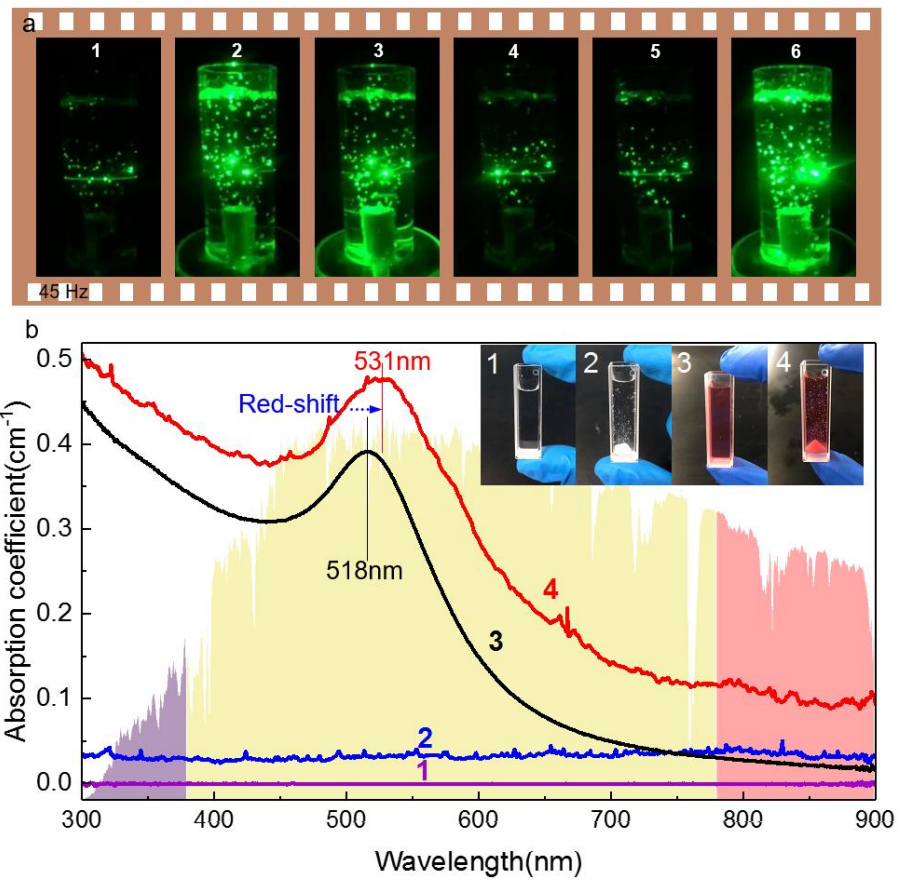

Fig. 3 The optical properties. a) Scattering properties of bubbly flow and b) absorption spectra of 1) pure water, 2) bubbly flow in pure water, 3) gold nanofluid and 4) gold nanofluid with bubbly flow.

The extinction properties of 1) pure water, 2) bubbly flow in pure water, 3) gold nanofluid and 4) gold nanofluid with bubbly flow are characterized using UV-vis spectroscopy (Fig. 3b). It can be seen that the gold nanofluid exhibits localized surface plasmon resonance (SPR), and the plasmon band is around $520 \mathrm{~nm}$. Such peak is consistent with the result of spherical Au NPs with a size of $16.5 \mathrm{~nm} .{ }^{[36]}$ The absorption spectrum of bubbly flow is observed with a parallel upshift and no obvious peak across the UV-visible range, indicating its semi-transparency to both UV and visible light due to its back-scattering or reflection properties. After incorporating the Au NPs within the bubbly flow, the plasmon band of the dynamic bubbly system of nanoparticleliquid mixture exhibits a slightly red-shifted absorbance band $(518 \rightarrow 531 \mathrm{~nm})$ of higher intensity at full wavelength (Fig. 3(b)4). This reflects that the peak absorption wavelength of the solution exhibiting SPR can be manipulated by the spontaneously particle adsorption on bubble surfaces. ${ }^{[38]}$ These interparticle couplings referred as hotspots allow electron transfer between particles, resulting in plasmonic oscillation at new characteristic frequencies. ${ }^{[20,39,40]}$ The dynamic assembling of particles at bubble interfaces thereby creates many different SPR modes based on the diverse 


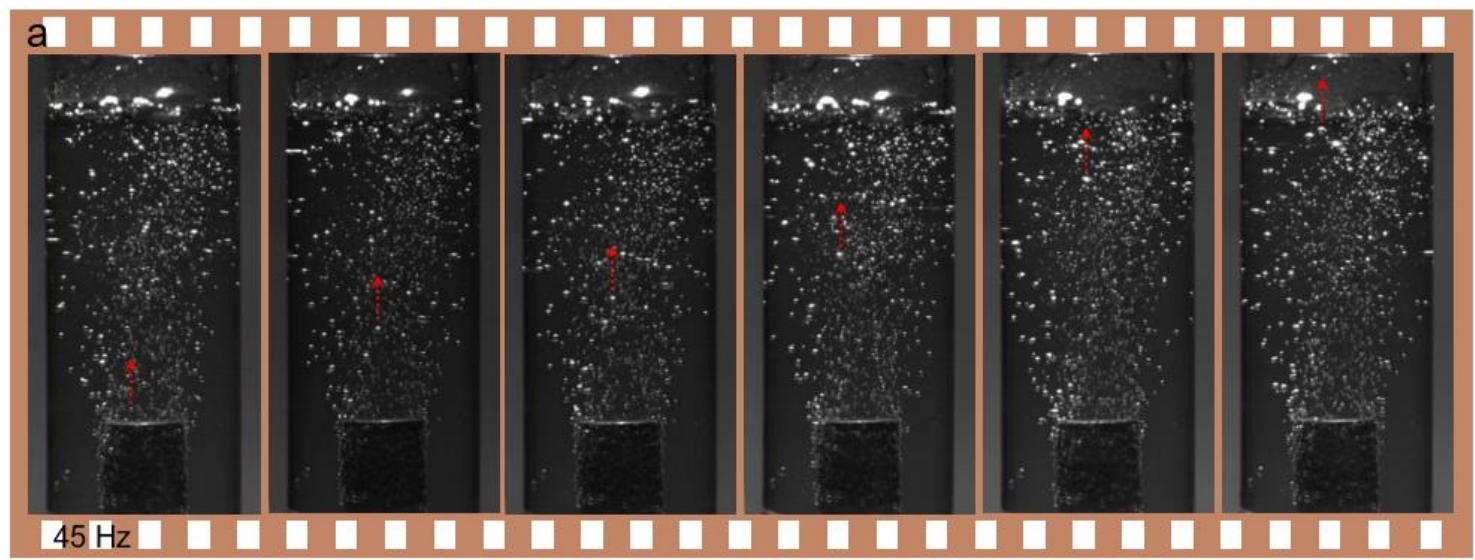

b
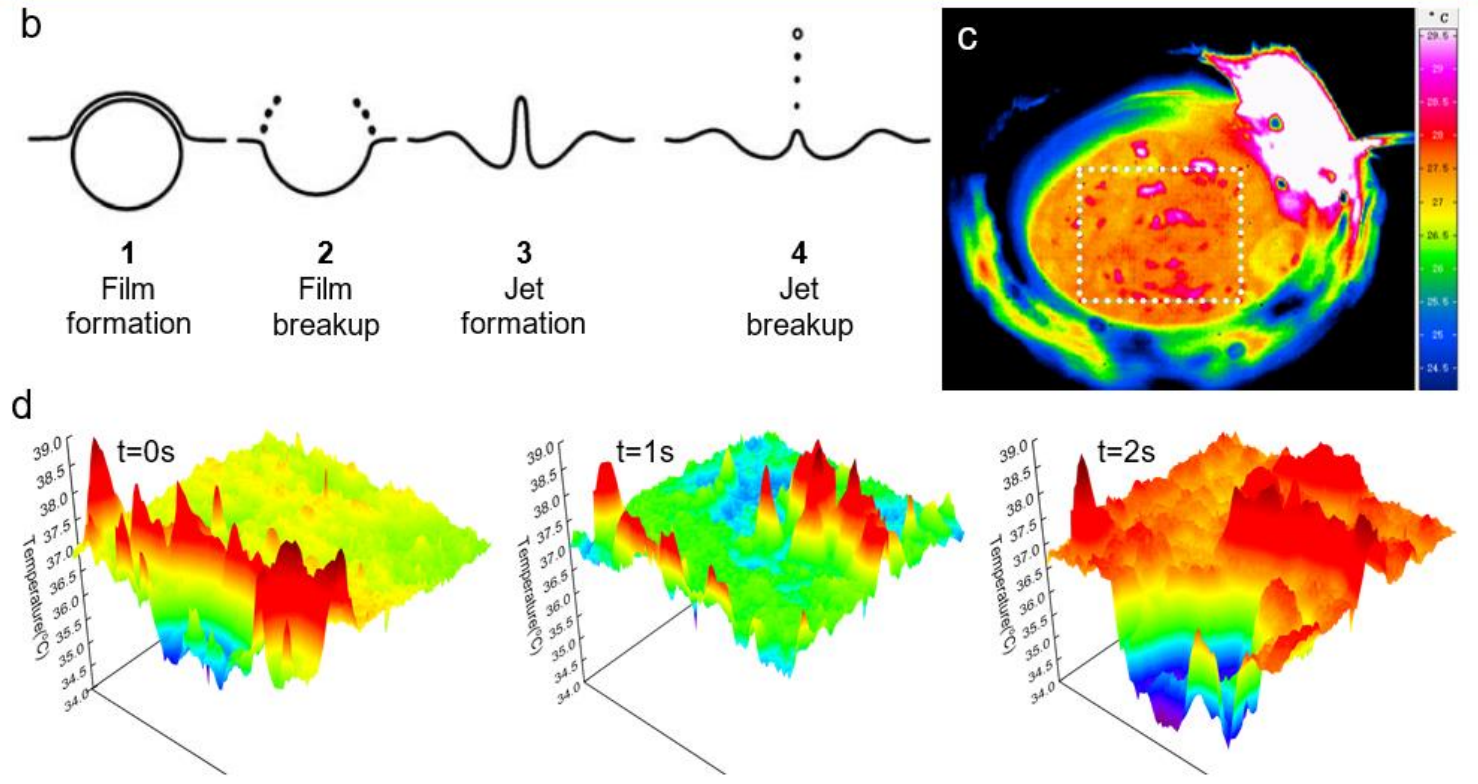

Fig. 4 The bubble dynamics during evaporation: a) Flow pattern evolution of gold nanofluid with bubbly flow, b) Stages of a bubble breaking through a free surface, c) An infrared image shows the nonuniform temperature at air-water interface. d) Temporal oscillations of temperature at air-water interface (the square area in Fig. c) during the bubble spray.

orientations and connections between particles. As such, the dynamic bubbly system involves distinct advantages: reducing void gaps between particles and improving light absorption by increasing the useful number of photons through the multi-scattering effect between bubbles. Due to different reflection and scattering properties in the multiphasic solutions, UV-vis spectroscopy may not be able to accurately capture the relative magnitude of absorption. But the absorption spectrum can be a rational analysis to qualitatively evaluate the optical properties.

\subsection{Bubble dynamics}

Key bubble dynamics occuring in the colum include bubble formation, bubble rising movement with water vapor capture and bubble spray boosting evaporation at air-water interface. The single bubble formation involves various forces. They are the upwards buoyancy force and a contact pressure force due to the action of overpressure inside the bubble, while the downward forces are the vertical component of the capillary force due to surface tension and the dynamic force due to inertial and viscous effects. The formation process is assumed in two stages. ${ }^{[29]}$ In the first stage, the bubble expands while its base remains on the tip of orifice, and that will stop when the sum of the upward forces equals to the sum of the downward forces. In the following stage, the upward forces exceed the sum of the downward forces and the bubble base moves away from the orifice, detaching from the sinter. The bubble growth and subsequent release from the orifice can be qualitatively described by the well-known Rayleigh-Plesset equation. ${ }^{[41]}$

$$
\rho_{l}\left(R \ddot{R}+\frac{3}{2} \dot{R}^{2}\right)=\nabla p_{\text {ext }}-\frac{2 \sigma}{R}-4 \mu_{l} \frac{\dot{R}}{R}
$$

Here $\sigma$ is the surface tension coefficient. $\rho_{l}$ and $\mu_{l}$ are the liquid density and viscosity, and dots denote time derivatives. $\nabla p_{\text {ext }}(t)$ and $R(t)$ refer to the time-varying gas pressure at the orifice exit relative to that in the liquid far away from the bubble and the radius of the forming bubble, respectively. The bubble formation described here provides basic 

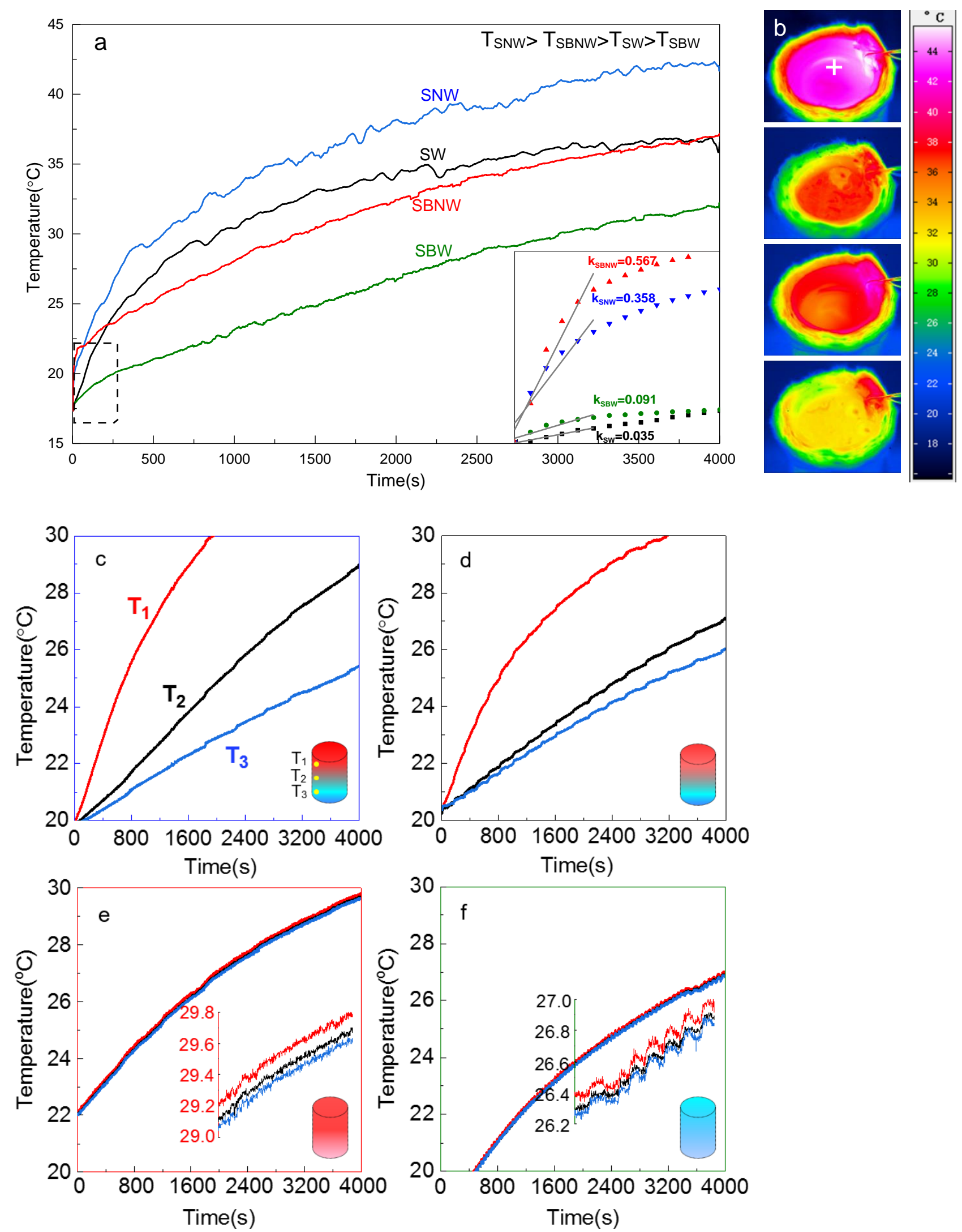

Fig. 5 a) Temporal history of interficial temperature in solar evaporation of gold nanofluid (SNW), water (SW), gold nanofluid with bubbly flow (SBNW) and water with bubbly flow (SBW). b) The infrared photos of interfaces at end of evaporation. c-f) Volumetric temperature distribution along colum depth in the corresponding cases. 
understanding on the massive generation of bubbles by blowing air through the porous sinter.

Fig. 4a shows the sequence images of rising bubbles within nanofluid for water vapor capture. The behavior of rising bubbles is complex and depends on the bubble size and shape, operating temperature and properties of the gas-slurry solution. In principle, bubble diameter $d_{b}$ is related to the pore size of sinter $d_{0}$, solution densities and air-liquid surface tension as given by $d_{b}=\left[\frac{6 \sigma d_{o}}{g\left(\rho_{l}-\rho_{a}\right)}\right]^{1 / 3}$. The bubble rise velocity governed by Newton's second law can be derived by equating the buoyant force on the bubble and the drag force. ${ }^{[42]}$ While adding Au NPs to water under irridation not only changes the liquid temperature and viscosity, but also introduces all known non-Newtonian phenomena, including shear and time dependence of the viscosity, and elastic effects such as stress relaxation and normal stress differences. ${ }^{[43]}$ As a result, with only few bubble coalescences observed at air-water surface, the multiphasic system results in a smaller bubble size with a better dispersion over the whole column compared with that of the bubbly flow in pure water. The motion of these highly dispersed bubbles ranging from 0.4 to $1.2 \mathrm{~mm}$ diameter is accompanied by a combination of trajectory oscillations (zig-zag or helical), and shape deformation oscillations (ellipsoidal) at a limited rise velocity. ${ }^{[4]}$ These oscillations both in shape and trajectory with a longer resident time thus enhance the rate, at which water vapor and heat transfer equilibrate within the bubbles. The saturation or equilibrium can attain in a few tenths of a second because of the oscillation flow inside bubbles due to shear forces acted on the bubble surface, which induces rapid heat and mass transfer within bubbles, much faster than that expected for quiescent diffusion. ${ }^{[29]}$

When the rising bubbles approaching to free surface,violent motions associated with the break through to gas would produce droplets that may persist in the gas stream to constitute a spray. ${ }^{[5]}$ The breakthrough details are surprisingly complicated (Fig. 4b). ${ }^{[46]}$ At first, a liquid film is formed on the top of bubble and the shattering of this film creates one set of droplets. These film droplets are mostly too small to be observed by the naked eye and their number per bubble bursting is still unknown. ${ }^{[47,48]}$ After breakthrough,the surface waves propagate inward as well as outward that induces an upward jet in the disruption center.The instability arising from the below of the collapsing bubble cavity causes the disintegration of this jet to form droplets. ${ }^{[49,50]}$ Generally, the size of jet droplets is substantially larger than the film droplets $(\sim \mu \mathrm{m})$, which is about a tenth the diameter of the original bubble. Hereby, the mass transfer from the lower liquid to the upper gas comes from both contributions of the disintegration of bubble cap and the fragmentation of upwardly directed liquid jet. Besides, the bubble spray also induces fast temperature oscillation and a typical infrared image with the nonuniform temperature at air-water interface is shown in Fig. 4c. The oscillation amplitude of temperature in spatial domain can be reached about $4{ }^{\circ} \mathrm{C}$ within millisecondes (Fig. 4d), which in turn leads to strong turbulent flow near the evaporation surface. ${ }^{[51]}$ This turbulent flow mixing with the upward gas flux from bubble bursting would carry a range of droplet sizes high into the atmosphere for an extending evaporation.

\subsection{Thermal properties}

The interfacial temperature evolutions of solar evaporation of gold nanofluid (SNW), water (SW), gold nanofluid with bubbly flow (SBNW) and water with bubbly flow (SBW)are compared to understand how the SBNW enhanced the water evaporation through heat concentration (Fig. 5a). At the begining, the SBNW heated up with a rising slope of 0.567 , which is much faster than the nanofluid case (0.358) as well as that of the other counterparts (see the inset of Fig. 5a). ${ }^{[8]}$ This can be explained from two aspects. First, the photothermal properties of SBNW facilitate more light to be converted into heat due to the multi-scattering effect brought about by bubbles, which amplifies the radiation flux to surronding $\mathrm{Au} \mathrm{NPs,}$ thus increasing the temperature at vaporation interfaces. ${ }^{[52]}$ On the other hand, the rising bubble swarm also serves as a foam thermos mug as that the system prevents the photothermal energy diffusion into around enviroment. ${ }^{[22,40,51]}$ After a few minutes of evaporation, heat generation in SBNW is partly brought away by the upward gas flux, as evidenced by a smaller rising slope, thereby exhibiting a lower interficial temperature than that of the SNW and SW. At the end of evaporation, the interfacial temperature in SBNW surpasses that of pure water again (Fig $5 \mathrm{a}$ and $\mathrm{b})$ due to the strong light absorption and heat equilibration over the whole column.

The corresponding results of bulk temperature distribution are shown in Fig. 5c-f. Three temperatures at top $\left(\mathrm{T}_{1}\right)$, middle $\left(\mathrm{T}_{2}\right)$ and bottom $\left(\mathrm{T}_{3}\right)$ of solution are traced simultaneously in each case to show the temperature difference along colum depth. It can be seen that the largest temperature gradient presents in the SNW case. This is due to the intense light absorption of Au NPs at the top portion of solution and the plasmonic heating is localized. A smaller temperature gradient is observed in pure water case because of the absorption nature of water for infrared light as well as the large aspect ratio of cloumn geometry (Fig. 5d). In contrast, the bubbly-flow solutions exhibit a nearly uniform bulk temperature in the cloumn since the fast heat equilibrium promoted by the oscillations arising from bubble formation and rising movement (Fig. 5e and f). The lightscattering bubbles coupling with the light absorptive nanoparticles concentrate the produced heat inside the bubble cloumn, giving rise to a bulk increasing of the temperature versus time. The final saturation temperature of SBNW is only about $5{ }^{\circ} \mathrm{C}$ lower than that of the SNW (Fig. 5a and e), indicating a volumetric heating attained in the cloumn. 
3.4. Steam generation performance
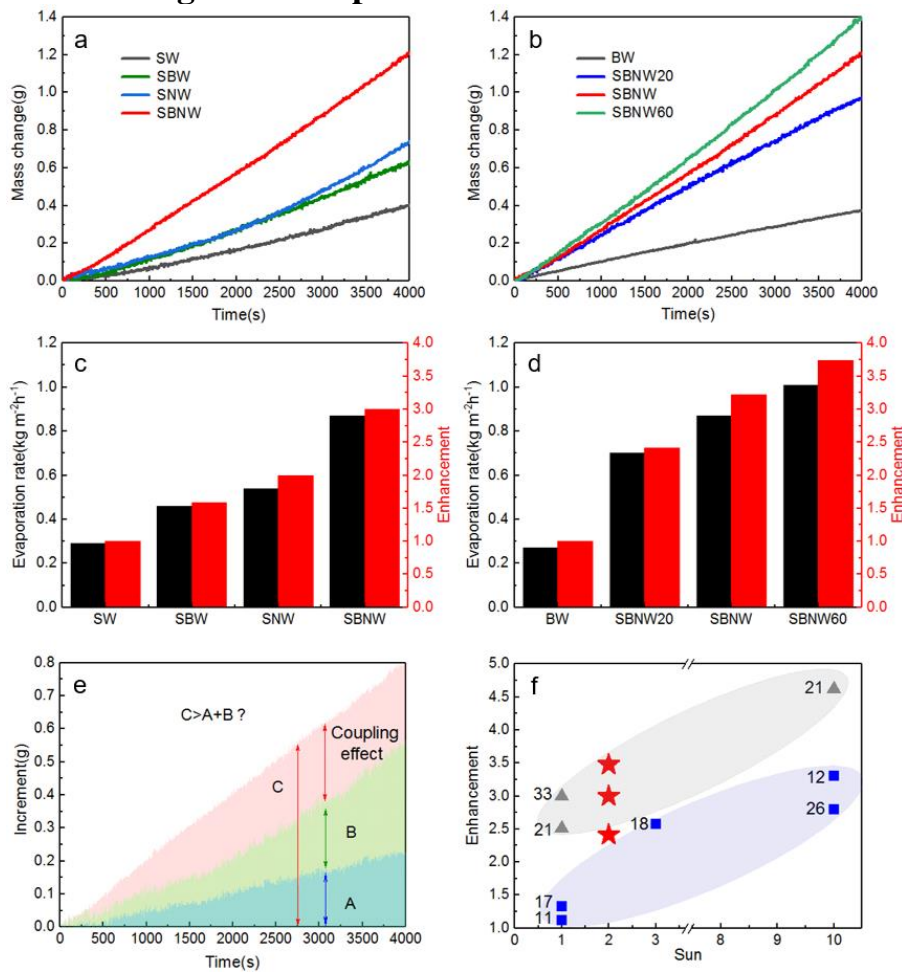

Fig. 6 Steam generation performance. a-b) transient mass change as a function of time. c-d) the corresponding evaporation rates and their enhancement. e) Physical mechnisms to steam generation, the steam increment from evaporation of SNW (A), SBW (B) and SBNW (C), respectively. f) Comparison of the enhancement as a function of solar irridiation for different systems in the references and this work. Data are shown for the multiphasic systems (red symbols), nanofluid systems (blue symbols) and interfacial systems (grey symbols), respectively

As a proof-of-concept to show the feasibility of implementation, we experimentally validated the steam generation performance in a fully functioning device. Their mass changes are compared at a long time scale $(0-4000 \mathrm{~s}$, Fig. 6a and b). Each of the cases is initiated at a relative steady-state to ensure that the solution temperature was consistent with the ambient temperature. Fig. 5a shows the mass changes as a function of time during the transient period for the SBNW, SNW, SBW and SW. The weight loss of water is found to increase near linearly with time.The SBNW evaporated the most water during the time scale $(\sim 1.21 \mathrm{~g})$, followed by the gold nanofluid $(\sim 0.74 \mathrm{~g})$, bubbly flow $(\sim 0.63 \mathrm{~g})$ and the base pure water $(\sim 0.40 \mathrm{~g})$. The transient evaporation rates of SBNW (dynamic slopes of the mass change line) are gradually increased within the first several minutes and remained stable during subsequent irradiation, which agrees well with the temperature increase profile. The mean evaporation rate of SBNW is $\sim 0.87 \mathrm{~kg} \mathrm{~m}^{-2}$ $\mathrm{h}^{-1}$, which is about 1.6 and 3.0 times enhancement than that of the nanofluid and pure water case, respectively (Fig. 6c).

The transient performance is related to how well dispersed the bubbles in the solutions are, because these bubbles act as a lamp deep in water to illuminate the surrounding particles. Hereby, nanofluids with welldispersed particles generate more heat closer to the bubble surfaces, and have a higher temperature and overall vaporation rate. In bubble column evaporator, the hydrodynamics, transport and mixing properties depend strongly on the prevailing flow regime. The upward movement of the bubble swarms can be characterized into three separate flow regimes. These regimes occur in order of increasing gas flow rate: Bubbly flow, churn turbulent flow and slug flow. In our experiment, we restricted the gas flow rate in the regime of bubbly flow for achieving more effective light scattering centers. As expected, the mass loss and evaporation rate of SBNW increase with the increase of the air flow rates since more bubbles offer more efficient light scattering centers to harvest solar energy and/or more interfaces for gas humidification (Fig. $6 \mathrm{~b}$ and d). For the bubbly flow at a gas flow rate of $40 \mathrm{~mL} / \mathrm{min}$ without light irradiation $(\mathrm{BW})$, its vaporation rate through humidification is about $0.27 \mathrm{~kg} \mathrm{~m}^{-2} \mathrm{~h}^{-1}$ that is quite similar to the evaporation of pure water $\left(0.29 \mathrm{~kg} \mathrm{~m}^{-2} \mathrm{~h}^{-1}\right)$. This evaporation rate mainly comes from the water vapour capture through bubble interfaces by the rising movement as well as the mass transport from subsequent bubble spray. In contrast, even at a small gas flow rate of $20 \mathrm{~mL} / \mathrm{min}$, the solar evaporation rate of SBNW can reach to $0.70 \mathrm{~kg} \mathrm{~m}^{-2} \mathrm{~h}^{-1}$ that is about 2.6 times larger than that of the bubbly-flow humidification. Such an enhancement is easily repeatable and more pronounced under larger gas flux, which are 3.2 and 3.7 times of the bubblyflow humidification at the gas flow rate of 40 and $60 \mathrm{~mL} / \mathrm{min}$, respectively. This marks that the evaporation rate of plasmon-bubble column can be easily improved above three times under solar irradiation.

\section{Discussion}

The experiments from optical, bubble dynamic, thermal and mass change aspect demonstrate that the particle-stabilized gas/liquid dispersion optimizes the contributions of light harvesting, heat management and mass transport to the overall device performance, leading to pronounced evaporation rates. To decouple the physical mechanisms underlying the phenomenon, we reexamine the contribution of light absorptive nanoparticles and light scattering bubbles after subtracting the base mass loss of SW from the mass loss curves (Fig. 6e). It is obvious that the steam increment from solar evaporation of $\operatorname{SBNW}(\mathrm{C})$ is larger than the sum of both contributions $(A+B)$, gaining a one plus one greater than two effect. This win-win game owes to the following factors: 1) the dispersed nanoparticles and bubbles functionally separated the light absorbtion and scattering to concentrate light energy into the mesoscale volume that maximizes photothermal conversion; 2) the dynamic bubbles not only serve as the scattering centers to amplify light energy, but also offer large interfacial areas to enhance mass transfer and act as foam insultor to reduce heat loss, thus fast raising the 
gas humidity; 3) the upward gas flux from bubble bursting accelerates the vapor generation due to the reduced pressure as well as the mass transport from film and jet droplets; and thus 4) the resulting light absorption and mass transport creating intense localized heating and efficient vaporization of the surrounding liquid, leading to the high performance evaporation.

To highlight the advantages of plasmon-assisted bubble column evaporator for steam generation, we present an overview of recently reported data, subdivided into three categories: nanofluid systems; interfacial systems; or multiphasic system. Fig. 6f compares the enhancement achieved with various systems. The typical enhancement of nanofluid system is below two, smaller than that of interfacial systems (three or above). While the enhancement of our multiphasic system is easily improved to above three without any complex material design and device optimization, even operation at a lower temperature. This performance outperforms the previous nanofluid system and is comparable to the today's prevalent interfacial systems. Additional advantages of the multiphasic system also include simplicity of operation, low operating costs and ease variation of the liquid residence time. ${ }^{[29]}$ Although it would be more reasonable to compare the absolute evaporation rate, often such data is not readily available due to different operation conditions, ${ }^{[5,6]}$ such as light intensity, solution properties, evaporator geometry, ambient air pressure, velocity, humidity, temperature, etc.

In sum, we proposed a spatial decoupling of light absorption and scattering concept to address the critical need for adaptive light absorption, thermal management and mass transport in solar steam generation. Demonstration with a plasmon-assisted bubble column evaporator shows that this method enhances the evaporation and steam generation rate by more than three fold through simply bubbling air into a gold nanofluid. Even larger enhancement should also be accessible through rational matching of the materials, size and concentration between bubbles and nanoparticles, or intentional increase of light intensity. This study points the way towards a simpler and more energy-efficient approach to solar steam generation in a wide range of climates, which is important for faster commercialization of solar steam technology, with potentially broad impacts on many applications such as residential water heating, desalination or wastewater treatment, catalytic slurry reactions, coal liquefaction, etc.

Further studies in physical aspects and engineering application of the plasmon-bubble column evaporator are still required. The controlled synthesis of nanomaterials, thoughtful device integration and novel system design are interesting aspects to be explored. To produce steam with high temperature and pressure through the column optimization are highly worthwile. Based on the spatial separation of optical absorption and scattering concept, developing a fully air-recycling evaporation system that links the evaporator and condensor would be significant for largescale application. However, the physical coupling or interaction between plasmonic heating and bubble dynamics is complex. Modelling of the bubbly flow of nanoparticleliquid mixtures remains challenging due to the difficult in formulating the modified bubble behaviors induced by the adsorbed nanoparticles. Nevertheless, particle-based methods such as Brownian dynamics and Mente Carlo simulations might provide insights into the embedded physics.

\section{Acknowledgements}

The research was supported by the Natural Science Foundation of China (52076077) and the Fundamental Research Funds for the Central Universities (2020DF002).

\section{Conflict of Interest}

There are no conflicts to declare.

\section{Support information}

Not applicable

\section{References}

[1] A. H. Cavusoglu, X. Chen, P. Gentine and O. Sahin, Nat. comm., 2017, 8, 617, doi: 10.1038/s41467-017-00581-w.

[2] K. A. Novick, D.L. Ficklin, P. C. Spoy, C. A. Williams, G. Bohrer, A.C. Oishi, S.A. Papuga, P. D. blanken, A. Noormets, B.N. Sulman, R.L. Scott, L. Wang and R. P. Phillips, Nat. Clim. Change, 2016, 6, 1023-1027, doi: 10.1038/nclimate3114.

[3] X. Chen and K. K. Tung, Science, 2014, 345, 897-903, doi: 10.1126/science. 1254937.

[4] S. Jasechko, Z. D. Sharp, J. J. Gibson, S. J. Birks, Y. Yi and P. J. Fawcett, Nature, 2013, 496, 347-350, doi: 10.1038/nature11983.

[5] P. Tao, G. Ni, C. Song, W. Shang, J. Wu, J. Zhu, G. Chen and T. Den, Nat. Energy, 2018, 3, 1031-1041, doi: 10.1038/s41560-018-0260-7.

[6] M. Gao, L. Zhu, C. K. Peh and G.W. Ho, Energ. Environ. Sci., 2019, 12, 841-864, doi: 10.1039/c8ee01146j.

[7] G.H.Liu, J.L.Xu and K.Y.Wang, Nano Energy, 2017, 41, 269-284, doi: 10.1016/j.nanoen.2017.09.005.

[8] J. Li, M. Du, G. Lv, L. Zhou, X. Li and L. Bertoluzzi, Adv. mater., 2018, 30, 1805159, doi: 10.1002/adma.201805159.

[9] L. Zhang, B. Tang, J. Wu, R. Li and P. Wang, Adv. mater., 2015, 27, 4889-4894, doi: 10.1002/adma.201502362.

[10] O. Neumann, C. Feronti, A. D. Neumann, A. Dong, K. Schell, B. Lu and P. Nordlander, PANS, 2013, 110, 11677-11681, doi: 10.1073/pnas.1310131110.

[11] X.Wang, Y. He, X. Liu, L. Shi and J. Zhu, Sol. Energy, 2017, 157, 35-46, doi: 10.1016/j.solener.2017.08.015.

[12] X. Wang, Y. He, G. Cheng, L. Shi, X. Liu and J. Zhu, Energ. Convers. Manage., 2016, 130, 176-183, doi: 10.1016/j.enconman.2016.10.049.

[13] M. Gao, P. K. N. Connor and G.W. Ho, Energy Environ. Sci., 2016, 9, 3151-3160, doi: 10.1039/c6ee00971a.

[14] A.R.Mallah, S.N.Kazi, M.N.M. Zubir and A.Badarudin, Appl. Energ., 2018, 229, 505-521, doi: 10.1016/j.apenergy.2018.07.113. 
[15] M. S. Zielinski, J. W. Choi, T.L. Grange, M. A. Modestino, S.M.H. Hashemi and Y. Pu, Nano Lett., 2016, 16, 2159-2167, doi: 10.1021/acs.nanolett.5b03901.

[16] S. Loeb, C. Li and J. H. Kim, Environ. Sci. Technol., 2018,52, 205-213, doi: 10.1021/acs.est.7b04442.

[17] Y. Fu, T. Mei, G. Wang, A. Guo, G. Dai and S. Wang, Appl. Therm. Eng., 2017, 114, 961-968, doi: 10.1016/j.applthermaleng.2016.12.054.

[18] Y. Fu, G. Wang, T. Mei, J. Li, J. Wang and X. Wang, ACS Sustain. Chem. Eng., 2017, 5, 4665-4671, doi: 10.1021/acssuschemeng.6b03207.

[19] N.J. Hogan, A. S. Urban, C. Ayala-Orozco, A. Pimpinelli, P Nordlander and N.J. Halas, Nano Lett., 2014, 14, 4640-4645, doi: $10.1021 / \mathrm{nl} 5016975$.

[20] L. Zhou, Y. Tan, J. Wang, W. Xu, Y. Yuan and W. Cai,Nat. Photonics, 2016, 10, 393-398, doi: 10.1038/nphoton.2016.75.

[21] H. Ghasemi, G. Ni, A.M. Marconnet, J. Loomis, S. Yerci and N. Miljkovic, Nat. Commun., 2014, 5, 4449, doi: 10.1038/ncomms5449.

[22] G.W. Ni, G. Li, S.V. Boriskina, H. Li and G. Chen, Nat. Energy, 2016, 1, 16126, doi: 10.1038/nenergy.2016.126.

[23] F. Zhao, X. Zhou, Y. Shi, X. Qian, M. Alexander and X. Zhao, Nat. Nanotechnol., 2018, 13, 489-495, doi: 10.1038/s41565-018-0097-z.

[24] K. Bae, G. Kang, S.K. Cho, W. Park, K. Kim and W.J. Padilla, Nat. Commun., 2015,6, 10103, doi: 10.1038/ncomms10103.

[25] W. Shang and T. Deng, Nat. Energy, 2016,9, 16133, doi: 10.1038/nenergy.2016.133.

[26] X. Z. Wang, Y. R. He, X. Liu, G. Cheng and J. Q. Zhu, Appl. Energ., 2017, 195, 414-425, doi: 10.1016/j.apenergy.2017.03.080.

[27] M.Taseidifar, M. Shahid and R. M. Pashley, Desalination, 2018, 432, 97-103, doi: 10.1016/j.desal.2018.01.003.

[28] A. Amaral, G. Bellandi, U. Rehman, R. Neves and I. Nopens, Water Res., 2018, 131, 346-355, doi: 10.1016/j.watres.2017.10.062.

[29] M. Shahid, C. Fan and R. M. Pashley, Int. Rev. Phys. Chem., 2016, 35, 143-185, doi: 10.1080/0144235X.2016.1147144.

[30] S. Nedeltchev, Chem. Eng. Sci., 2017, 157, 169-181, doi: 10.1016/j.ces.2016.06.047.

[31] H. M. Abd-ur-Rehman and F. A. Al-Sulaiman, Energ. Convers. Manage., 2016, 127, 667-678, doi: 10.1016/j.enconman.2016.09.053.

[32] H. L. Penman, P. Roy. Soc. A-MATHPhy., 1948, 193, 120 145, doi: 10.1098/rspa.1948.0037.

[33] F. Liu, B. Zhao, W. Wu, H. Yang, Y. Ning and Y. Lai, $A d v$. Funct. Mater, 2018, 28, 1803266, doi: 10.1002/adfm.201803266.
[34] P. J. C. Kuiper, H. Veenman \& Zonen, 1961.

[35] C. Finnerty, L. Zhang, D. L. Sedlak, K. L. Nelson and B. Mi, Energy Environ. Sci., 2017,51, 11701-11709, doi: 10.1021/acs.est.7b03040.

[36] G. H. Liu, H. Cao and J. L. Xu, Sol. Energy, 2018, 170, 184-191, doi: 10.1016/j.solener.2018.05.069.

[37] G. E. Davis, J. Opt. Soc. Am., 1955, 45, 572.

[38] Y.Yuan, X.Li and J.Tu, Int. J. Heat Mass Tran., 2018, 120, 552-567, doi: 10.1016/j.ijheatmasstransfer.2017.12.053.

[39] N. Jiang, X. Zhuo and J. Wang, Chem. Rev., 2018, 118, 3054-3099, doi: 10.1021/acs.chemrev.7b00252.

[40] J. Chen, J. Feng, Z. Li, P. Xu, X. Wang, W. Yin and Y. Yin, Nano Lett., 2019, 19, 400-407, doi: 10.1021/acs.nanolett.8b04157.

[41] J. Rodríguez-Rodríguez, A. Sevilla, C. Martínez-Bazán and J. M. Gordillo, Annu. Rev. Fluid Mech., 2015, 47, 405-429, doi: 10.1146/annurev-fluid-010814-014658.

[42] P. R. Ranjitha, R. Ratheesh, J. S. Jayakumar and S. Balakrishnan, Iop. Conf. Ser-Mat. Sci., 2018, 310, 012065, doi: 10.1088/1757-899X/310/1/012065.

[43] E. W. Llewellin, H. M. Mader and S. D. R. Wilson, P. Roy. Soc. A-Math. Phy., 2002, 458, 987-1016, doi: 10.1098/rspa.2001.0924.

[44] I. Leifer, R. Patro and P. Bowyer, J. Atmos. Ocean. Tech., 2000, 17, 1392-1402, doi: 10.1175/15200426(2000)017<1392:ASOTTV>2.0.CO;2.

[45] F. Knelman, N. Dombrowski and D. M. Newitt, Nature, 1954, 173, 261, doi: 10.1038/173261a0.

[46] C. E. Brennen, Cambridge University Press, 2005.

[47] J. Feng, M. Roch é, D. Vigolo, L.N. Arnaudov, S.D. Stoyanov, T.D. Gurkov and H.A. Stone, Nat. Phys., 2014,10, 606-612, doi: 10.1038/nphys3003.

[48] J. C. Bird, R.D. Ruiter, L. Courbin and H. A. Stone, Nature, 2010,465, 759-762, doi: 10.1038/nature09069.

[49] C. Y. Lai,J. Eggers and L. Deike, Phys. Rev. Lett., 2018,121, 144501, doi: 10.1103/PhysRevLett.121.144501.

[50] L. Deike, E. Ghabache, L.B. Gérard, A.K. Das and S. Thomas, Phys. Rev. Fluids, 2018, 3, 013603, doi: 10.1103/PhysRevFluids.3.013603.

[51] G. Ni, S.H. Zandavi, S.M. Javid, S.V. Boriskina, T.A. Cooper and G. Chen, Energy Environ. Sci., 2018, 11, 1510-1519, doi: 10.1039/c8ee00220g.

[52] D. Zhao, H. Duan, S. Yu, Y. Zhang, J. He, X. Quan and T. Deng, Sci. Rep., 2015, 5, 17276, doi: 10.1038/srep17276.

Publisher's Note: Engineered Science Publisher remains neutral with regard to jurisdictional claims in published maps and institutional affiliations. 